\title{
EFECTO DEL CHOQUE TÉRMICO SOBRE LA EFICIENCIA EN LA TRIPLOIDIZACIÓN Y LA SOBREVIVENCIA DE LARVAS DE Rhamdia quelen
}

\section{EFFECT OF THERMALSHOCK ON TRIPLOIDIZATION EFFICIENCY AND SURVIVAL OF LARVAE OF Rhamdia quelen}

\begin{abstract}
Liliana Cardona $^{1}$, Martha Olivera $^{2}$, Mónica Botero $^{3}$, Ariel Tarazona ${ }^{4}$
${ }^{1}$ Zoot. M.Sc. Facultad de Ciencias Agropecuarias, Dpto. Producción Animal, grupo BIOGEM, Universidad Nacional de Colombia, Sede Medellín, Colombia. Calle 56A \# 63-20 Bloque 50 Of 316, Medellín, Antioquia, Imcardonb@unal.edu.co; 2 DMV, Dra. Sci. Agr. Grupo de Investigación BIOGENESIS/Universidad de Antioquia-Universidad Nacional de Colombia. Carrera 75 \# 65-87 Bloque 46-202, Medellín Antioquia, syngamia@gmail.com; ${ }^{3}$ Zoot. Ph.D. GRICA/Universidad de Antioquia, Colombia. Carrera 75 \# 65-87, Medellín, Antioquia, vicedecanaturafca@gmail.com; ${ }^{4}$ Zoot. M.Sc, c.PhD. Facultad de Ciencias Agropecuarias, Dpto. Producción Animal, Universidad Nacional de Colombia, Sede Medellín, Colombia. Calle 56A \# 63-20 Bloque 50 Of 316, Medellín, Antioquia. amtarazonam@unal.edu.co
\end{abstract}

Rev. U.D.C.A Act. \& Div. Cient. 15(2): 409 - 417, 2012

\section{RESUMEN}

La triploidía permite mejoras en el cultivo de peces, ya que se evita la madurez sexual, controla la superpoblación y aumenta la estabilidad en el desarrollo. Debido a su esterilidad, se pueden retrasar las cosechas, logrando peces de mayor peso y edad. El objetivo del estudio fue evaluar el efecto del choque térmico sobre la eficiencia en la triploidización y la sobrevivencia de larvas de Rhamdia quelen. Se emplearon dos hembras y tres machos sexualmente maduros, inducidos con extracto de hipófisis de carpa. Las ovas fueron inseminadas y siete minutos posfertilización, sometidas a choque térmico, con tres temperaturas: 34,36 y $38^{\circ} \mathrm{C}$ y dos tiempos: 2 y 5 minutos. Posteriormente, fueron incubadas hasta el momento de la eclosión. Se registraron: temperatura, oxígeno y porcentajes de eclosión y de sobrevivencia. Se evaluó la ploidía de las larvas, mediante tinción con nitrato de plata de las regiones organizadoras de nucléolo (RON). El porcentaje de eclosión fue igual en el control y en los TTOs de 2 minutos; todos los TTOs de 5 minutos presentaron menor porcentaje de eclosión $(\mathrm{p}<0.05)$. El porcentaje de sobrevivencia fue mayor al $91 \%$ en todos los tratamientos, sin diferencia estadística entre ellos. Los porcentajes de triploidía para los tratamientos con choque térmico, sin incluir el control, oscilaron entre 78,8 y $96,6 \%$, sin observarse diferencia significativa entre ellos ( $p>0.05)$. Se concluye, que el choque térmico meiótico, con las tres temperaturas y con una duración de 2 y 5 minutos, induce la triploidía en Rhamdia quelen.
Palabras clave: Desarrollo embrionario, meiosis, región organizadora de nucléolo, triploide.

\section{SUMMARY}

Triploidy enables improvements in fish farming since it avoids sexual maturity, controls overpopulation and increases the developmental stability. Because of its sterility, the fish harvest may be delayed, attaining fishes with greater weight and age. The aim of this study was to evaluate the effect of thermal shock on the efficiency of triploidization and the survival of Rhamdia quelen larvae. Two females and three males sexually mature were used, induced with carp pituitary extract. The eggs were inseminated and seven minutes postfertilization, submitted to thermal shock with three temperatures: 34,36 and $38^{\circ} \mathrm{C}$ during two and five minutes, respectively; then incubated until hatching. Temperature, oxygen, hatching rates and survival were recorded. Larval ploidy was evaluated by staining organizing nucleolus regions (NOR) with silver nitrate. The hatching rate was similar in the control and the treatments, being of two minutes, while hatching percentage o was lower $(p<0.05)$ in the five minutes treatments. The survival rate was higher than $91 \%$ in all treatments, without statistical difference between them. The percentages of triploidy by heat shock treatments, not including the control, ranged between 78.8 and $96.6 \%$, without significant difference between them ( $p>0.05)$. It was concluded that meiotic thermal shock with the three temperatures and duration within 2 to 5 minutes can induce triploid in Rhamdia quelen. 
Key words: Embryo development, meiosis, nucleolus organizer region, triploid.

\section{INTRODUCCIÓN}

Algunas características de los peces, como la fecundación externa, la alta fecundidad, el control de la diferenciación sexual y un intervalo generacional relativamente corto (Tabata, 2008), los convierten en ejemplares deseables para la manipulación sexual y cromosómica (Thorgaard, 1983). La biotecnología ha hecho muchos aportes en diferentes ciencias y disciplinas, entre ellas, la acuicultura (Pineda et al. 2004); un avance importante ha sido la obtención de individuos poliploides, con una variación cuantitativa del conjunto de cromosomas (Díaz \& Neira, 2005), la cual, ha permitido mejoras en la producción de especies de cultivo, ya que se evita la madurez sexual (Piferrer et al. 2009; Díaz \& Neira, 2005), se controla la superpoblación y aumenta la estabilidad en el desarrollo (Piferrer et al. 2009); dada la condición de esterilidad, se pueden retrasar las cosechas, logrando peces de mayor peso y edad (Díaz \& Neira, 2005). Adicionalmente, pueden servir para programas de repoblamiento sin que exista competencia reproductiva con las especies nativas ( $\mathrm{Pi}$ ferrer et al. 2009). Algunos de los cambios y consecuencias de la tecnología fueron citados por Benfey (1999), dentro de los que se destacan una disminución en la relación área/ volumen de células de tejidos y de órganos, puesto que estas poseen un mayor contenido de material genético, que conlleva a que se afecten fenómenos de comunicación celular y transporte, a través de membranas. Aunque la biotecnología para la obtención de individuos triploides se ha implementado desde 1943 en diversas familias de peces (Hulata, 2001), aún es poco conocido cómo se origina y se mantiene esta condición de triploidía (Benfey, 1999) y surgen en ellos una serie de cambios que no han permitido mejorar el uso de estas biotecnologías y entender el comportamiento y las respuestas fisiológicas de los organismos al medio. Se cree que pueden existir otras alteraciones que involucran la forma de la célula, la fluidez de la membrana celular y la síntesis de ácidos nucleicos, cuando se utilizan choques de temperatura, para inhibir la expulsión del segundo cuerpo polar (Hildebrandt, 2002). Estos choques han sido uno de los métodos más empleados para la obtención de triploides a gran escala (Piferrer et al. 2009; Díaz \& Neira, 2005; Thorgaard, 1983; Pineda et al. 2004).

Rhamdia quelen (Quoy \& Gaimard, 1824) (Pisces: Heptapteridae) es un pez perteneciente al orden Siluriforme, que ha demostrado buena resistencia a la manipulación (Rodríguez, 2007) y es de fácil adaptación y reproducción, bajo condiciones de laboratorio, por lo que se ha convertido en un modelo para realizar investigaciones en diferentes áreas, dentro de las cuales, la biotecnología aplicada es un campo de impacto productivo en Colombia y adaptable a las especies de producción más utilizadas (Pineda et al. 2004) o en aquellas que se consideran promisorias para el desarrollo piscícola del país. El objetivo de este trabajo fue evaluar el efecto del choque térmico sobre la eficiencia en la triploidización y la sobrevivencia de larvas de $R h$. quelen.

\section{MATERIALES Y MÉTODOS}

Animales: Se emplearon dos hembras de 0,1219 y de $0,1776 \mathrm{~kg}$ y tres machos de 0,$0977 ; 0,081$ y $0,1099 \mathrm{~kg}$, sexualmente maduros, seleccionados según las características descritas por Varela et al (1982). Los animales fueron obtenidos de ejemplares en cautiverio, mantenidos en la Estación Piscícola San José del Nus, ubicada en el municipio de San Roque (Antioquia); la temperatura promedio del agua fue de $24^{\circ} \mathrm{C}$. Los animales fueron transportados hasta el laboratorio, donde permanecieron en acuarios durante un periodo de adaptación de 20 días.

Obtención de gametos: Para la inducción de la reproducción, se utilizó extracto de hipófisis de carpa (EHC, Stoller Fisheries, USA), usando el protocolo propuesto por Woynarovich \& Horvath (1983), con las modificaciones descritas a continuación. Para las hembras, se aplicaron dos dosis de 0,5 y $5,0 \mathrm{mg} / \mathrm{kg}$ de peso vivo, con intervalo de doce horas, entre la primera y la segunda dosis. Los machos fueron inducidos con una dosis única de $3,5 \mathrm{mg} / \mathrm{kg}$, la cual, se aplicó simultáneamente con la segunda dosis de la hembra. Los animales fueron previamente anestesiados con Quinaldine ${ }^{\circledR}$ $200 \mu \mathrm{L} / 3 \mathrm{~L}$ de agua y se emplearon los protocolos de manipulación de menor estrés para los animales. Los gametos, se obtuvieron por extrusión manual en dirección cráneo-caudal y se mezclaron para formar un grupo de oocitos y un grupo de semen; de este último, se tomó una muestra de $0,5 \mathrm{~mL}$, para evaluar parámetros espermáticos y asegurar la calidad del mismo; dentro de éstos, se tuvo en cuenta movilidad (Araújo et al. 2003), tiempo de activación y concentración espermática (Cruz-Casallas et al. 2005).

Choque térmico meiótico: Las ovas fueron inseminadas para permitir la fertilización; posteriormente, se hidrataron con agua declorada a $25^{\circ} \mathrm{C}$; se depositó una muestra de $2 \mathrm{~mL}$ para cada tratamiento en incubadoras; 7 minutos posfertilización y se sometieron a choque térmico. Los tratamientos de choque térmico evaluados fueron a tres temperaturas: 34,36 y $38^{\circ} \mathrm{C}$, cada una con dos tiempos: 2 y 5 minutos (34-2, 34-5, 36-2, 36-5, 38-2, 38-5); para lo cual, todas las incubadoras fueron sumergidas, simultáneamente, en baños termostáticos, durante tiempos y temperaturas respectivas mencionadas. El grupo control consistió en ovas inseminadas, a una temperatura de $25^{\circ} \mathrm{C}$ y no se les hizo choque térmico. Cada tratamiento, incluyendo el control, tuvo cuatro réplicas, excepto los de $38^{\circ} \mathrm{C}$, que se hicieron por duplicado, debido al número de ovocitos disponibles. Una vez finaliza- 
do el choque térmico, las ovas de cada réplica fueron distribuidas de manera aleatoria en incubadoras de acrílico tipo californiana, con agua a $25^{\circ} \mathrm{C}$ y se mantuvieron allí hasta el momento de la eclosión. Durante este tiempo, se registraron los siguientes parámetros de calidad de agua: temperatura y oxígeno, evaluados mediante un oxímetro Orion 3 Star DO y pH con un pHmetro marca WTW.

Evaluación del porcentaje de eclosión y sobrevivencia: Para determinar los porcentajes de eclosión (a) y de sobrevivencia (b), se utilizaron las siguientes fórmulas:

$$
\begin{gathered}
\% \text { Eclosión }=\left(\frac{\sum \text { larvas vivas y muertas }}{\sum \text { larvas vivas, muertas y huevos }}\right) * 100 \\
\% \text { sobrervivencia de larvas con vitelo }=\left(\frac{\sum \text { larvas vivas }}{\sum \text { larvas totales }}\right) * 100
\end{gathered}
$$

Las larvas vivas fueron separadas por réplicas en frascos de vidrio, con $2 \mathrm{~L}$ de agua, previamente declorada y mantenidas a $25^{\circ} \mathrm{C}$.

Evaluación del porcentaje de triploidía: Nueve días después de la eclosión, se tomó al azar una muestra de poslarvas de cada réplica, de acuerdo al número de sobrevivientes a la fecha y se anestesiaron con Quinaldine $\AA$, en una proporción de $50 \mu \mathrm{L} / 0,25$ litros de agua. Se obtuvieron suspensiones celulares individuales de cada larva, empleando el protocolo reportado por Vozzi et al. (2003), utilizando citrato de sodio al 0,7\%, como solución hipotónica, en vez de cloruro de potasio. Posteriormente, se trataron con solución de metanol: ácido acético 3:1 y se adicionó ácido acético al 50\%, durante 3 minutos. Se realizó goteo en placas portaobjetos, incluyendo los restos de los animales, los cuales, se maceraron. Las placas se secaron sobre una plancha térmica a $60^{\circ} \mathrm{C}$.

Para facilitar la observación de núcleos interfásicos, cada placa fue coloreada durante 10 minutos, con tinción de Giemsa al 5\%, preparada en fresco y llevadas a la plancha térmica. Se realizó tinción con nitrato de plata de las regiones organizadoras de nucléolo (RON), según el protocolo descrito por Alonso et al. (2000). La observación de los núcleos interfásicos con la expresión de las regiones organizadoras de nucléolo, se hizo en un microscopio óptico marca Leica (40X - 100X). Se contabilizó un número mínimo de 50 núcleos por placa y se determinó, en cada núcleo observado, el número de regiones organizadoras de nucléolo expresadas. Un individuo se consideró triploide, si presentaba al menos un núcleo con 3 RONes.

Análisis estadístico: Se usó un diseño de clasificación experimental completamente aleatorizado efecto fijo balanceado en arreglo factorial $3 \times 2$, con una adición correspondiente al control; se realizó análisis suplementado con ANOVAS de tipo unidimensional y pruebas de contraste de Tukey, con un error tipo I del 5\%. Las variables, el porcentaje de eclosión y de sobrevivencia, se transformaron con base en la función arco seno. Se aplicó análisis descriptivo exploratorio de tipo unidimensional, con el objetivo de hallar promedio, desviación típica y coeficiente de variación, para cada una de las variables de interés; para los análisis, se empleó el programa SAS versión 8.2.

\section{RESULTADOS Y DISCUSIÓN}

El promedio de temperatura registrado en el experimento fue de $25 \pm 0,9^{\circ} \mathrm{C}$; el valor mínimo de oxígeno disuelto en las diferentes réplicas fue de $4,8 \mathrm{mg} / \mathrm{L}$ y el mayor de $6,1 \mathrm{mg} / \mathrm{L}$; los valores de $\mathrm{pH}$ oscilaron entre 7,13 $\pm 0,20$ y 7,22 $\pm 0,10$. Respecto a los parámetros físico-químicos del agua y su posible influencia en los resultados obtenidos para las variables reproductivas evaluadas en los diferentes tratamientos durante el tiempo de incubación y eclosión, estuvieron dentro de los rangos permisibles y recomendados para especies de aguas cálidas (Roldán, 1992), lo que es apoyado por los valores encontrados, dado que no hubo diferencia significativa entre tratamientos.

Eclosión: El mayor porcentaje de eclosión se obtuvo en el tratamiento control a una temperatura de $25^{\circ} \mathrm{C}$ con un valor de $43,5 \pm 3,3 \%$ sin choque térmico. El valor mínimo correspondiente a 15,8 $\pm 10,4 \%$ fue obtenido en el tratamiento 36-5. En general, se observa que los menores porcentajes de eclosión se presentan cuando el tiempo de duración del choque térmico es mayor ( 5 minutos). En la figura $1 \mathrm{~A}$, se presentan los resultados obtenidos para el porcentaje de eclosión de ovas, sometidas a choque térmico meiótico.

El porcentaje de eclosión presentó diferencia significativa $(p<0,05)$ entre el control y los tratamientos 34-5, 38-5 y 365 ; los resultados de los tratamientos con tiempos de choque térmico de 2 minutos, no presentaron diferencia significativa $(p>0,05)$ (Figura 1A). La tabla 1 muestra los promedios, las desviaciones típicas, los coeficientes de variación y la diferencia estadística de los porcentajes de eclosión, luego de someter las ovas a los diferentes tratamientos.

En cuanto a los tratamientos, se podría decir que el tiempo del choque térmico tiene un efecto deletéreo para este parámetro. Se observa una marcada tendencia de obtener menores porcentajes de eclosión con los tratamientos de choque de 5 minutos, al oscilar entre 15,8 a 17,8\% (Tabla 1), siendo éstos los más bajos.

Las células en los distintos organismos tienen diferentes respuestas cuando existe incremento en el estrés, causado por una elevación de la temperatura, que depende de la duración 


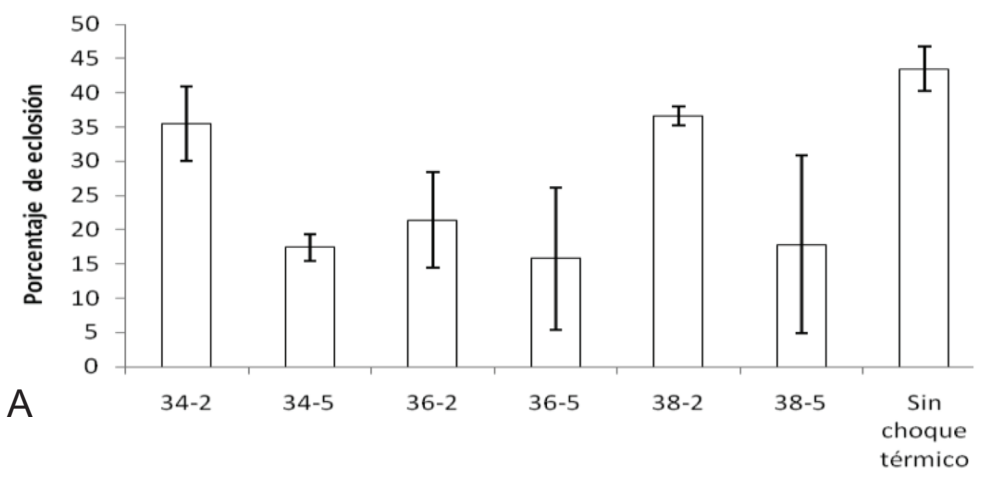

Tratamiento temperatura - tiempo

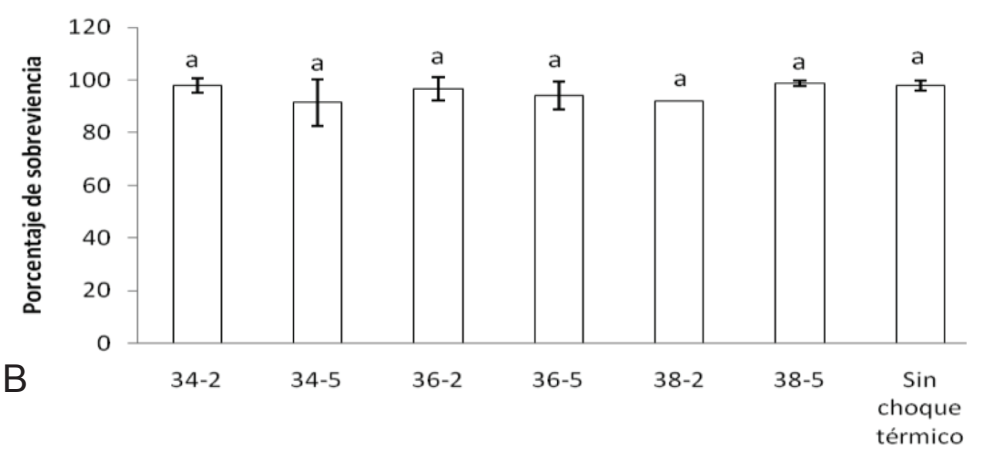

Tratamiento temperatura - tiempo

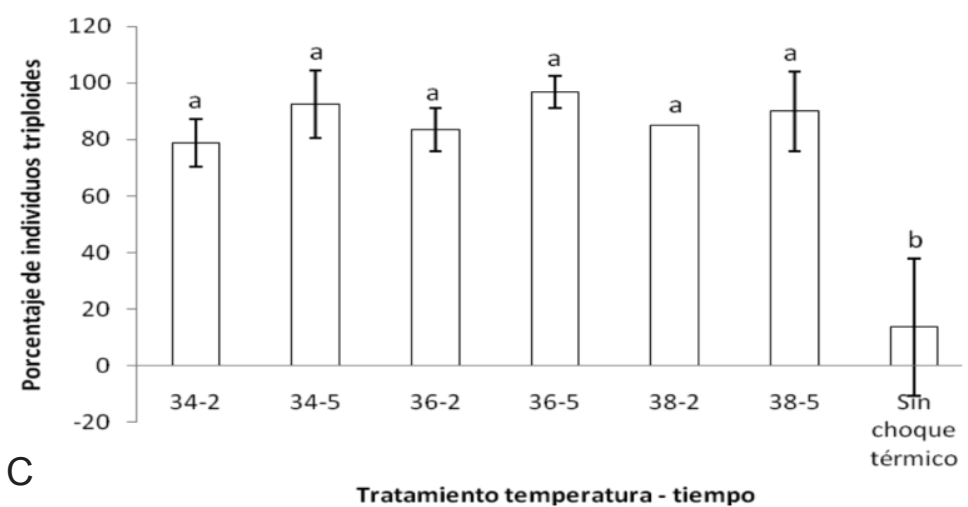

Figura 1. A) Porcentaje de eclosión de ovas de Rhamdiaquelen sometidas a choque térmico; B) Porcentaje de sobrevivencia por tratamientos (promedio \pm d.e). Letras diferentes significan diferencia estadística $(p<0.05)$ y C) Porcentaje de triploidía por tratamientos (promedio \pm d.e). Letras diferentes significan diferencia estadística $(p<0.05)$.

que esta permanezca alta (Arvelo, 2002). Cuando es leve, como en el caso del choque con duración de dos minutos, se podría aseverar, aunque no fue demostrado en este trabajo, que se presenta una superproducción de proteínas de choque térmico (Hsp), que permiten que se desarrolle la termo tolerancia, previniendo la apoptosis al desencadenar mecanismos protectores contra la oxidación, como los reportados por Fleury et al. (2002). Si se presenta una situación de estrés más intenso, como ocurrió con el choque de cinco minutos, se puede generar necrosis celular (Arvelo, 2002), fenómeno que se ve reflejado en las bajas tasas de eclosión, en ovas sometidas a este tratamiento.

Sobrevivencia: El porcentaje de sobrevivencia fue alto, superando el $91 \%$ en todos los tratamientos, siendo muy homogéneos los resultados, de acuerdo a lo observado en la desviación estándar (Figura 1B).

No se encontraron diferencias significativas entre los tratamientos 34-2, 34-5, 36-2, 36-5, 38-2, $38-5$ ( $p>0,05$ ). Los 
Tabla 1. Porcentaje de eclosión, triploidía y descripción del número de núcleos interfásicos con 1 RON, 2 RON y 3 RON observados por tratamiento, expresado como promedio \pm d.e.

\begin{tabular}{|c|c|c|c|c|c|c|c|}
\hline TTO & $\mathrm{n}$ & \% de eclosión & C.V & $1 \mathrm{RON}$ & $2 \mathrm{RON}$ & $3 \mathrm{RON}$ & \% Triploidía \\
\hline $34-2$ & 80 & $35,5 \pm 5,4^{a b}$ & 15,2 & $26,5 \pm 6,0^{a b}$ & $26,3 \pm 5,2^{a}$ & $1,8 \pm 1,7^{\mathrm{c}}$ & $78,75 \pm 8,53^{a}$ \\
\hline $34-5$ & 78 & $17,4 \pm 2,0^{b}$ & 11,6 & $25,5 \pm 5,7^{a b c}$ & $26,9 \pm 5,4^{a}$ & $2,2 \pm 1,6^{b c}$ & $92,5 \pm 11,9^{a}$ \\
\hline $36-2$ & 73 & $2, .4 \pm 7,0^{a b}$ & 32,7 & $24,7 \pm 5,5^{b c}$ & $28,4 \pm 4,5^{a}$ & $2,2 \pm 1,4^{b c}$ & $83,3 \pm 7,6^{a}$ \\
\hline $36-5$ & 37 & $15,8 \pm 10,4^{b}$ & 65,9 & $26,1 \pm 5,7^{a b c}$ & $25,4 \pm 6,6^{a}$ & $4,8 \pm 3,7^{a}$ & $96,6 \pm 5,7^{a}$ \\
\hline $38-2$ & 39 & $36,6 \pm 1,4^{a b}$ & 3,8 & $22,6 \pm 5,6^{c}$ & $27,9 \pm 5,2^{a}$ & $3,0 \pm 2,5^{b}$ & $85 \pm 0,0^{a}$ \\
\hline $38-5$ & 21 & $17,8 \pm 13,0^{b}$ & 73,2 & $26,6 \pm 5,1^{a b}$ & $28,7 \pm 4,6^{a}$ & $1,5 \pm 1,1^{\mathrm{c}}$ & $90 \pm 14,1^{\mathrm{a}}$ \\
\hline Sin choque & 64 & $43,5 \pm 3,3^{a}$ & 7,7 & $29,2 \pm 8,4^{a}$ & $26,1 \pm 8,3^{a}$ & $0,1 \pm 0,8^{d}$ & $13,7 \pm 24,2^{b}$ \\
\hline
\end{tabular}

Letras diferentes significan diferencia estadística ( $<$ <.05). Desviación estándar: d.e.; coeficiente de variación: cv.

valores expresados en promedio \pm desviación estándar y el coeficiente de variación para los tratamientos y sin choque térmico, fueron $97,9 \pm 2,6$ (c.v=2,6), $91,5 \pm 8,8$ (c.v=9,6), $96,6 \pm 4,3$ (c.v=4,4), 94,1 $\pm 5,1(\mathrm{c} . \mathrm{v}=5,5), 92,0 \pm 0,0$ (c.v $=0,0), 98,8 \pm 1,0$ (c.v=1,1), 97,9 $\pm 1,7$ (c.v=1,7), respectivamente.

Vozzi et al. (2003) encontraron, en el momento de la eclosión, el mayor porcentaje de sobrevivencia, correspondiente a $33,40 \%$, cuando se sometieron ovas de Rh. quelen a choque térmico, a $36^{\circ} \mathrm{C}$, durante 5 minutos, 5 minutos posfertilización; valor inferior a los reportados en el presente estudio. La misma especie, sometida a choques térmicos fríos, para obtención de triploides, exhibió $65,4 \pm 3,1 \%$ de sobrevivencia (Soller et al. 2007).

Después de la eclosión, no se observaron diferencias significativas en la sobrevivencia de larvas entre los tratamientos. Lo anterior conduce a suponer que no existe efecto del choque térmico en las larvas, luego de la eclosión y durante la reabsorción de saco vitelino. Es posible que ello sea mediado por una familia de proteínas de choque térmico (Hsp); moléculas encargadas de la respuesta celular al estrés, que se sintetizan en forma constitutiva e inducible, una vez sobreviene aquel y actúan como chaperonas moleculares asociadas con ensamble y plegamiento de otras proteínas (Hartl, 1996). Se ha reportado que algunas de las proteínas de este tipo persisten en las células, cuando desaparece el factor causante del estrés (Dubeau et al. 1998).

Chippari et al. (1999) reportaron que el rango de confort para $R h$. quelen, en el periodo de larvicultura, es de $21-30^{\circ} \mathrm{C}$, valores de temperatura que abarcan la mantenida durante el experimento, por lo tanto, las condiciones de temperatura en las que se desarrollaron las larvas no habrían sido las causantes de su muerte.

Ploidía: Para determinar la distribución de las regiones organizadoras de nucléolo en núcleos interfásicos de suspensiones celulares, se realizó un Anova, encontrándose diferencia significativa $(p<0,05)$ en el análisis de la variable número de núcleos interfásicos, con 1 RON; entre el control respecto al tratamiento 36-2 y al 38-2; el tratamiento 38-5 respecto al 38-2 y tratamiento 34-2 respecto al 38-2. El mayor número de núcleos con 1 RON, se presentó en el control, mientras que el menor se observó en el 38-2. La menor desviación estándar encontrada para la expresión de una Región Organizadora de Nucléolo, se evidenció en el tratamiento 38-5 (Tabla 1).

Para la variable número de núcleos con 2 RON, no hubo diferencia significativa $(p>0,05)$. De manera general, se observa que el número de núcleos con 2 RON fue mayor al encontrado con 1 RON (Tabla 1). Dado que las Regiones Organizadoras de Nucléolo son requeridas por las células somáticas para la construcción de los ribosomas, posiblemente, como respuesta de la célula a los choques de temperatura, se aumentaría la capacidad de síntesis de proteínas, principalmente, las de choque térmico, que se ve reflejado en el número de estas estructuras presentes en el núcleo, como mecanismo para evitar la muerte celular.

En los núcleos interfásicos de individuos considerados triploides de los diferentes tratamientos, los cuales, exhibieron $3 \mathrm{RON}$, se encontraron diferencias significativas $(p<0,05)$. Hubo diferencia entre el tratamiento 36-5 y los demás; en el tratamiento 38-2 con respecto al 38-5, al control y al 34-2; en el tratamiento 34-5, 36-2, 34-2 y 34-5 respecto al control. 
La frecuencia de presentación de $3 \mathrm{RON}$ fue inferior que la de 1 y 2 RON (Tabla 1$)$.

Las frecuencias mostradas anteriormente corresponden a individuos diploides y triploides, siendo estos últimos los que exhibieron hasta 3 RON, por lo tanto, en todos los tratamientos, se presentó poliploidía.

En cuanto a la distribución de las regiones organizadoras de nucléolo en núcleos interfásicos de suspensiones celulares, donde se encontraron diferencias a las temperaturas y tiempos de choque térmico respecto a 1 RON o dos RONes, se podría deber a que las variaciones de RONes se pueden incrementar por recombinación homóloga desigual dentro del ribosoma del gen, tal como lo sugieren Schmickel et al. (1985) y que es altamente dependiente de la actividad celular y que dependen de los depósitos en los centros fibrilares o en la segregación de los nucléolos durante la interfase en las RONes durante la mitosis (Hernández et al. 1980).

La figura 2 muestra la expresión de RONes en núcleos interfásicos de poslarvas diploides y triploides de Rh. quelen.

Los porcentajes de triploidía para los tratamientos con choque térmico, sin incluir el control, oscilaron entre 78,8 y $96,6 \%$, sin observarse diferencia significativa entre ellos ( $p>0,05$ ), siendo menor para el tratamiento 34-2 y el mayor para 36-5. Con respecto al tratamiento sin choque térmico, se hallaron valores de $13,7 \%$ de triploides, observándose diferencia estadística $(p<0,05)$, con respecto a los demás tratamientos (Tabla 1); adicionalmente, se observa una amplia desviación estándar (Figura 1C).

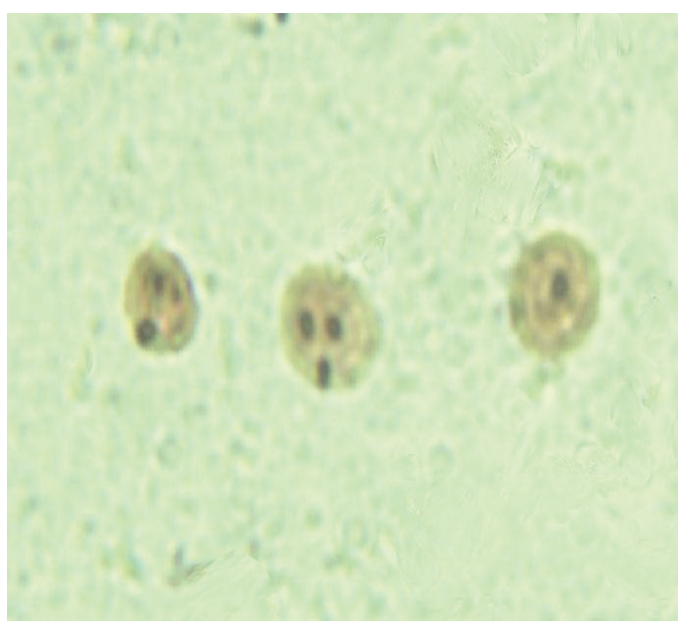

La eficiencia en la triploidización encontrada en el presente estudio, no es un parámetro definitivo a la hora de escoger una temperatura y un tiempo de choque térmico para obtener individuos triploides en esta especie, ya que se deben tener en cuenta otros factores, como las tasas de eclosión y la sobrevivencia, que son afectados por los procedimientos realizados. Lincoln \& Bye (1984) reportaron para trucha arco iris (Oncorhynchus mykiss) que, en la medida en que se aumenta la temperatura para choque térmico, se aumenta el número de triploides, hasta cierto punto, pero se afecta negativamente el porcentaje de sobrevivencia en la fase de ojos visibles y, aún más, el de vesícula reabsorbida.

Soller et al. (2007) realizaron inducción de triploidía en $R h$. quelen con choque térmico frío; el mayor porcentaje de triploides obtenido fue $97,9 \%$ con tratamiento a una temperatura de $4^{\circ} \mathrm{C}$, durante 20 minutos; valor muy cercano al obtenido al hacerlo a $36^{\circ} \mathrm{C}, 5$ minutos $(96,6 \%)$, en este trabajo. Con la realización de choque térmico cálido a $36^{\circ} \mathrm{C}$, durante cinco minutos, 5 minutos posteriores a la fertilización, Vozzi et al. (2003), para la misma especie, obtuvieron $74,8 \%$ de triploides; un dato inferior al encontrado en este trabajo, variando únicamente el momento del choque. Aunque en el trabajo de Vozzi no evaluaron exactamente 7 minutos, como tiempo posfertilización para hacer el choque, es posible que entre este tiempo y 5 minutos no existan diferencias y lo que realmente determinó un inferior porcentaje de triploidía fue el procedimiento o las herramientas utilizadas, para que la distribución del calor fuera uniforme en las unidades experimentales y se manifestara el verdadero efecto de la temperatura y el tiempo. Se puede hipotetizar que las diferencias existentes pueden ser originadas por los contrastes presentes, entre la $R h$. quelen procedente de las lagunas marginales, en

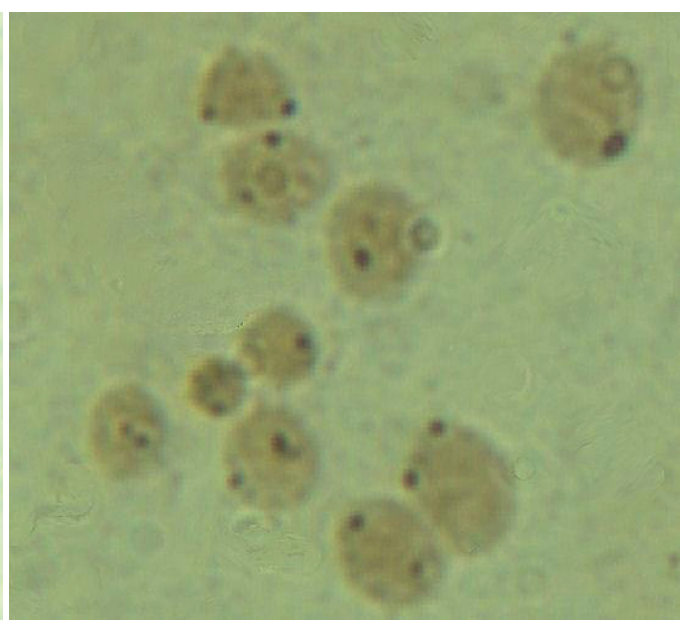

Figura 2. Expresión de RONes en núcleos interfásicos de poslarvas diploides y triploides de Rhamdia quelen teñidos con nitrato de plata. A la izquierda, se observa núcleo con 3 regiones organizadoras de nucléolo, indicando triploidía (40X). A la derecha, núcleos con 1 y 2 regiones organizadoras, pertenecientes a un individuo diploide (40X). 
la provincia de Corrientes, en Argentina y la procedente de la cuenca la Vega, en el municipio de San Roque, en Colombia y las condiciones ambientales en las que se encuentran y, es por ello, que se hace necesario la estandarización de los procedimientos, para la obtención de individuos poliploides, para cada población en particular.

El $100 \%$ de individuos triploides se ha obtenido en Salmo salar, por medio de choque térmico (Benfey \& Sutterlin, 1984); en Rh. quelen este mismo porcentaje se ha alcanzado, realizando un choque de presión (Huergo \& ZaniboniFilho, 2006), lo que conlleva a resultados más consistentes, pero presenta la desventaja de requerir equipos costosos (Maxime, 2008). En Colombia, la obtención de triploides de $R h$. quelen en porcentajes superiores al $90 \%$ es un gran logro, ya que se tienen pocos reportes, hasta el momento, y la estandarización de ese procedimiento es importante para cuando se quieran utilizar dichos individuos como modelos experimentales o se quieran aprovechar sus ventajas de esterilidad; además, serviría para promover a los productores acuícolas del país a utilizar este tipo de biotecnologías, que son de gran importancia, para obtener ventajas productivas y son económicas.

En el grupo en que no se realizó choque térmico, se halló un porcentaje de triploides equivalente al $13,7 \%$, lo que conlleva suponer varias posibilidades: primero, que de manera natural, en una pequeña proporción, se haya presentado una falla en la extrusión del cuerpo polar después de fertilizar los huevos (Tiwary et al. 2004); o se dio el proceso de fertilización normal, pero los huevos posiblemente por estrés mecánico generado en la manipulación; o por agentes químicos, se encontraban en un estado no reducido 2n (Borin et al. 2002).

Phillips et al. (1986) propusieron el método de identificación de individuos triploides por medio de la observación de regiones organizadoras de nucléolo teñidas con plata, en núcleos interfásicos, en diferentes especies de salmónidos, incluyendo la trucha arco iris; sin embargo, sugirió que la técnica solamente debía ser usada en el caso único de que la especie expresara un cromosoma con una región organizadora de nucléolo por genoma haploide. Zharskaya \& Zatsepina (2007) plantearon que dentro del cariotipo existen unos cromosomas específicos, en los cuales, se encuentra el conjunto de regiones organizadoras de nucléolo y de ADNr y el número presente de cromosomas de este tipo, está directamente relacionado con el número de nucléolos en la interfase, que puede ser alterado como respuesta celular al choque térmico. En $R$ h. quelen se ha reportado que en cada cromosoma submetacéntrico del par número 20 se encuentran ubicadas las RONes (Moraes et al. 2007); sin embargo, existen otros autores que las han reportado en pares acrocéntricos (Fenocchio et al. 2003) y subtelocéntricos (Stivari et al. 2004), en una frecuencia mucho menor; Moraes et al.
(2007) afirman que la variación en la ubicación de las regiones organizadoras de nucléolo asociadas a cromosomas es debido a rearreglos cromosómicos; no obstante, estas variaciones no alteran su número y, por ello, no limitan la utilización de la técnica para la identificación de la ploidía en la especie.

El choque térmico meiótico con las temperaturas 34, 36 y $38^{\circ} \mathrm{C}$ y con una duración de 2 y 5 minutos permite obtener individuos triploides en Rhamdia quelen; sin embargo, es importante considerar que existe un efecto marcado en el tiempo de duración del choque, que disminuye los porcentajes de eclosión, por lo tanto, es deseable usar el menor tiempo para el choque de temperatura con el que se puedan obtener triploides, observándose que el tratamiento 38-2 si bien no arrojó los mayores porcentajes de triplodía es el que mejor se comporta en cuanto a tasas de eclosión y sobrevivencia.

Este trabajo es el primero que se reporta en el país en cuanto a obtención de triploides en una especie nativa, lo que es importante, dado que teniéndose un referente para su producción, se pueden emplear estos ejemplares como modelos experimentales para estudios en otras áreas o para aprovechar su condición de esterilidad y no madurez sexual, que favorece mayores ganancias de peso en etapas específicas de crecimiento y, además, serviría para promover a los productores acuícolas del país a utilizar este tipo de biotecnologías altamente importantes para diversificar producto, ya que permitiría ampliar el mercado del filete en ciertas especies y son de bajo costo.

Agradecimientos: A Colciencias y a la Universidad Nacional Sede Medellín, por financiar el programa de Jóvenes Investigadores 2008-2009, en el marco del convenio especial de cooperación No 209. Al laboratorio de Larvicultura de peces y producción de alimento vivo (Larpeali), de la Facultad de Ciencias Agrarias de la Universidad de Antioquia. Conflicto de intereses: El manuscrito fue preparado y revisado con la participación de todos los autores, quienes declaramos que no existe ningún conflicto de intereses que ponga en riesgo la validez de los resultados presentados.

\section{BIBLIOGRAFÍA}

1. ALONSO, M.; TABATA, Y.; RIGOLINO, M.; TSUKAMOTO, R. 2000. Effect of induced triploidy on fin regeneration of juvenile rainbow trout, Oncorhynchus mykiss. J. Exp. Zool .(Brasil). 287:493-502.

2. ARVELO, F. 2002. Mitocondria y apoptosis. Acta Cient. Ven. 53:297-306. 
3. ARAÚJO, H.; CORDERO, W.; RUGELES, C.; ATENCIO, V. 2003. Evaluación de las características seminales de Blanquillo Sorubim cuspicaudus inducido con ovaprim ${ }^{\circledR}$. Rev. Col. Cienc. Pec. 16 (suplemento):78p.

4. BENFEY, T. 1999. The physiology and behavior of triploid fishes. Rev. Fish. Sci. (Inglaterra). 7(1):39-67.

5. BENFEY, T.; SUTTERLIN, A. 1984. Triploidy induced by heat shock and hydrostatic pressure in landlocked Atlantic salmon (Salmo salar L.). Aquacult (EE.UU). 42:359-367.

6. BORIN, L.; MARTINS, I.; OLIVEIRA, C. 2002. A natural triploid in Trichomycterus davisi (Siluriformes, Trichomycteridae): mitotic and meiotic characterization by chromosome banding and synaptonemal complex analyses. Genetica (EE.UU). 115:253-258.

7. CHIPPARI, A.; GOMES, L.; BALDISSEROTTO, B. 1999. Lethal temperature for silver catfish, Rhamdia quelen, fingerlings. J. Appl. Aquac. (Brasil). 9(4):11-21.

8. CRUZ-CASALLAS, P.E.; LOMBO-RODRÍGUEZ, D.A.; VELASCO-SANTAMARÍA, Y.M. 2005. Milt quality and spermatozoa morphology of captive Brycon siebenthalae (Eigenmann) broodstock. Aquacult. Res. 36:682-86.

9. DÍAZ, N.; NEIRA, R. 2005. Biotecnología aplicada a la acuicultura I. Biotecnologías clásicas aplicadas a la reproducción de especies cultivadas. Cie. Invest. Agraria (Chile). 32(1):45-59.

10. DUBEAU, S.; FENG, P.; TREMBLAY, G.; BRADLEY, T. 1998. Thermal shock of salmon in vivo induces the heat shock protein hsp 70 and confers protection against osmotic shock. Aquacult. 168:311-323.

11. FENOCCHIO, A.S.; BERTOLLO, L.A.C.; TAKAHASHI, C.S.; DIAS, A.L.; SWARÇA, A.C. 2003. Cytogenetic studies and correlated considerations on Rhamdiinae relationships (Pisces, Siluroidei, Pimelodidae). Cytologia. 68(4):363-368.

12. FLEURY, C.; MIGNOTE, B.; VAYSSIERE, J. 2002. Mitochondrial reactive oxygen species in cell death signaling. Biochimie (EE.UU). 84:131-141.

13. HARTL, F. 1996. Molecular chaperones in cellular protein folding. Nature. 381:571-580.

14. HERNÁNDEZ, D.; HUBERT, J.; BOURGEOIS, C. 1980. Ultrastructural localization of Ag-NOR stained pro- teins in the nucleolus during the cell cycle and in other nucleolar structures. Chromosoma (Inglaterra). 79: 42-362.

15. HILDEBRANDT, B.; WUST, P.; ALHERS, O.; DIEING, A.; SREENIVASA, G.; KERNER, T.; FELIX, R.; RIESS, H. 2002. The cellular and molecular basis of hyperthermia. Crit. Rev. Oncol. Hematol. (EE.UU). 43:33-56.

16. HUERGO, G.; ZANIBONI, E. 2006. Triploidy induction in JundiáRhamdiaquelen through hydrostatic pressure shock. J. Appl. Aquacult. 18(4):45-57.

17. HULATA, G. 2001. Genetics manipulations in aquaculture: a rewiew of stock improvement by classical and modern technologies. Genetica (EE.UU). 111:155173.

18. LINCOLN, R.; BYE, V. 1984. Triploid rainbows show commercial potential. Fish Farmer (EE.UU). 7:3032.

19. MAXIME, V. 2008. The physiology of triploid fish: current knowledge and comparisons with diploid fish. Fish Fisheries (Inglaterra). 9:67-78.

20. MORAES, V.; CEREALI, S.; FROEHLICH, O.; DIAS, A. 2007. Cytogenetic characterization of Rhamdia quelen (Siluriformes, Heptapteridae) from the Bodoquena Plateau, MatoGrosso do Sul, Brazil. Genetics Mol. Res. (Brasil).6(3):627-633.

21. PHILLIPS, R.; ZAJICEK, J.; IHSSEN, P.; JOHNSON, O. 1986. Application of silver staining to the identification of triploid fish cells. Aquacult. 54:313-319.

22. PIFERRER, F.; BEAUMONT, A.; FALGUIÈRE, J.C.; FLAJŠHANS, M.; HAFFRAY, P.; COLOMBO, L. 2009. Polyploid fish and shellfish: Production, biology and applications to aquaculture for performance improvement and genetic containment. Aquacult. 293(34):125-156.

23. PINEDA, H.; JARAMILLO, J.; ECHEVERRI, M.; OLIVERA, A. 2004. Triploidía en trucha arcoiris (Oncorhynchus mykiss): posibilidades en Colombia. Rev. Col. Cienc. Pec. 17(1):45-52.

24. RODRÍGUEZ, M. 2007. Fichas de especies. Acuicultura Uruguay. Disponible desde Internet en: http://www. dinara.gub.uy/fichas\%20de\%20especies/bagre\%20 negro.htm (con acceso 14/08/07). 
25. ROLDÁN, G. 1992. Fundamentos de Limnología Neotroropical. Edit. Universidad de Antioquia, Medellín. 529p.

26. SCHMICKEL, R.; GONZALEZ, I.; ERICKSON, J. 1985. Nucleolus organizing genes on chromosome 21: recombination and non-disjunction. Ann. N.Y. Acad. Sci. 450:121-131.

27. SOLLER, F.; GUIMARÃES, R.; OROZCO, R.; SILVA, A. 2007. Triploidy induction by cold shock in the South American catfish, Rhamdia quelen (Siluriformes) (Quoy\&Gaimard, 1824). Aquacult. 272S1:S110S114.

28. STIVARI, M.; MARTINS-SANTOS, I. 2004. Karyotype diversity in two populations of Rhamdia quelen (Pisces, Heptapteridade). Cytologia (Japón). 69:25-34.

29. TABATA, Y. 2008. Biotecnologías aplicadas a truticultura. Rev. Col. Cienc. Pec. 21:455-522.

30. THORGAARD, G. 1983. Chromosome set manipulation and sex control. En: Hoard, W.S.; Randall, D.J.; Donaldson, E.M. (eds). Fish Physiology. Acad. Press. New York. p.405-434.
31. TIWARY, B.; KIRUBAGARAN, R.; RAY, A. 2004. The biology of triploid fish. Rev. Fish Biol. Fisheries (ReinoUnido). 14:391-402.

32. VARELA, Z.; FISCHER, K.; FABIANO, G. 1982. Reproducción artificial de bagre negro Rhamdia sapo. Project reports. Montevideo. 34p. Disponible desde Internet en: http://www.fao.org/docrep/field/003/ AC568S/AC568S01.htm (con acceso 15/08/2007).

33. VOZZI, A.; SÁNCHEZ, S.; PERMINGEAT, E. 2003. Inducción de triploidía en Rhamdia quelen (PISCES, PIMELODIDAE). Bol. Inst. Pesca, São Paulo. 29(1):8794.

34. WOYNAROVICH, E.; HORVÁTH, L. 1983. A propagação artificial de peixes de águas tropicais. Brasilia: FAO/ CODEVASF/CNPq. 220p.

35. ZHARSKAYA, O.; ZATSEPINA, O. 2007. The dynamics and mechanisms of nucleolar reorganization during mitosis. Cell Tissue Biol. (EE.UU). 1(4):277-292.

Recibido: Enero 12 de 2012

Aceptado: Octubre 26 de 2012 